\title{
Flow cytometry sorting of nuclei enables the first global characterization of Paramecium germline DNA and transposable elements
}

Frédéric Guérin ${ }^{1}$, Olivier Arnaiz², Nicole Boggetto ${ }^{1}$ Cyril Denby Wilkes ${ }^{2,3}$, Eric Meyer ${ }^{4}$, Linda Sperling ${ }^{2}$ and Sandra Duharcourt ${ }^{1 *}$

\begin{abstract}
Background: DNA elimination is developmentally programmed in a wide variety of eukaryotes, including unicellular ciliates, and leads to the generation of distinct germline and somatic genomes. The ciliate Paramecium tetraurelia harbors two types of nuclei with different functions and genome structures. The transcriptionally inactive micronucleus contains the complete germline genome, while the somatic macronucleus contains a reduced genome streamlined for gene expression. During development of the somatic macronucleus, the germline genome undergoes massive and reproducible DNA elimination events. Availability of both the somatic and germline genomes is essential to examine the genome changes that occur during programmed DNA elimination and ultimately decipher the mechanisms underlying the specific removal of germline-limited sequences.

Results: We developed a novel experimental approach that uses flow cell imaging and flow cytometry to sort subpopulations of nuclei to high purity. We sorted vegetative micronuclei and macronuclei during development of $P$. tetraurelia. We validated the method by flow cell imaging and by high throughput DNA sequencing. Our work establishes the proof of principle that developing somatic macronuclei can be sorted from a complex biological sample to high purity based on their size, shape and DNA content. This method enabled us to sequence, for the first time, the germline DNA from pure micronuclei and to identify novel transposable elements. Sequencing the germline DNA confirms that the Pgm domesticated transposase is required for the excision of all $\sim 45,000$ Internal Eliminated Sequences. Comparison of the germline DNA and unrearranged DNA obtained from PGM-silenced cells reveals that the latter does not provide a faithful representation of the germline genome.

Conclusions: We developed a flow cytometry-based method to purify P. tetraurelia nuclei to high purity and provided quality control with flow cell imaging and high throughput DNA sequencing. We identified 61 germline transposable elements including the first Paramecium retrotransposons. This approach paves the way to sequence the germline genomes of $P$. aurelia sibling species for future comparative genomic studies.
\end{abstract}

Keywords: Flow cytometry, Non-LTR retrotransposons, ITm DNA transposons, Programmed DNA elimination, High throughput sequencing

\footnotetext{
* Correspondence: sandra.duharcourt@ijm.fr

${ }^{1}$ Institut Jacques Monod, CNRS, UMR 7592, Université Paris Diderot, Sorbonne

Paris Cité, Paris F-75205, France

Full list of author information is available at the end of the article
} 


\section{Background}

Major genome changes can occur during somatic differentiation. In diverse organisms, programmed DNA elimination leads to the removal of specific-germline DNA sequences during development of somatic cells and thus generates germline and somatic genomes with distinct architectures. This process has been described in a wide variety of animals and in ciliates, suggesting that it has likely arisen independently in different lineages [1]. Ciliates are unicellular eukaryotes with separate germline and somatic nuclei. In the ciliate Paramecium tetraurelia, two small, genetically identical diploid micronuclei (MIC, $2 \mathrm{n}, \sim 3 \mu \mathrm{m})$ contain the germline genome that is transmitted to sexual progeny after meiosis. A large, transcriptionally active somatic macronucleus (MAC, 800n, $\sim 30 \mu \mathrm{m}$ ) contains a reduced genome streamlined for gene expression. At each sexual cycle, the parental MAC is lost, while new MICs and MACs, destined for the progeny, develop from a copy of the diploid zygotic nucleus. In the new developing MAC, the germline genome is endoreplicated to reach its final ploidy of $\sim 800 \mathrm{n}$ and undergoes massive programmed DNA elimination (for review [2]) (Fig. 1). Large DNA regions containing transposable elements and other repeated sequences are eliminated, leading to chromosome breakage and de novo telomere addition. In addition, 45,000 short, unique, Internal Eliminated Sequences (IESs) are precisely excised. At least $25 \%$ of the $\sim 100 \mathrm{Mb}$ MIC genome is removed [3]. The distinctive genome architectures of ciliates make them attractive model systems to study the complex mechanisms underlying programmed DNA elimination. Meiosisspecific small RNA and chromatin modification pathways, similar to those found in plants and animals for the formation of heterochromatin and silencing of repeated sequences, are involved in the epigenetic programming of DNA elimination $[4,5]$.
Comprehensive description of genome changes that occur during programmed DNA elimination requires comparison of the germline and the somatic genomes. While the rearranged somatic MAC genome was sequenced and assembled 10 years ago allowing gene annotation [6], technical difficulties in obtaining pure MIC DNA (0.5\% of total genomic DNA) have long been a major obstacle to sequencing the germline genome of P. tetraurelia. Pioneering work used Percoll gradient centrifugation to separate MICs from MACs [7]. Despite high bacterial contamination of the resultant MIC DNA [3], this led to the discovery of germline-limited sequences [7, 8]. More recently, DNA enriched in unrearranged germline-like sequences was obtained from cells RNAi-depleted of PiggyMac (Pgm), the domesticated transposase required for developmental genome rearrangements [9]. Deep-sequencing of this DNA (PGM DNA) enabled genome wide-characterization of 45,000 IESs in P. tetraurelia [3]. However, how faithfully PGM DNA mimics the true germline genome found in the $\mathrm{MIC}$ remains an open question.

We report here a new and reliable method to purify MICs involving a critical step of flow cytometry. The method also allows isolation of developing MACs. Complete separation of nuclei was validated by flow cell imaging and by high throughput DNA sequencing. We show that PGM DNA is in fact not equivalent to MIC DNA. Contigs assembled from the MIC DNA allowed discovery of new $P$. tetraurelia transposable element families.

\section{Results and Discussion}

\section{Purification of new developing MACs}

Before tackling the purification of the tiny MICs, we decided to purify new developing MACs from cells undergoing the sexual process of autogamy (self-fertilization) (Fig. 1). At each sexual cycle, the parental MAC

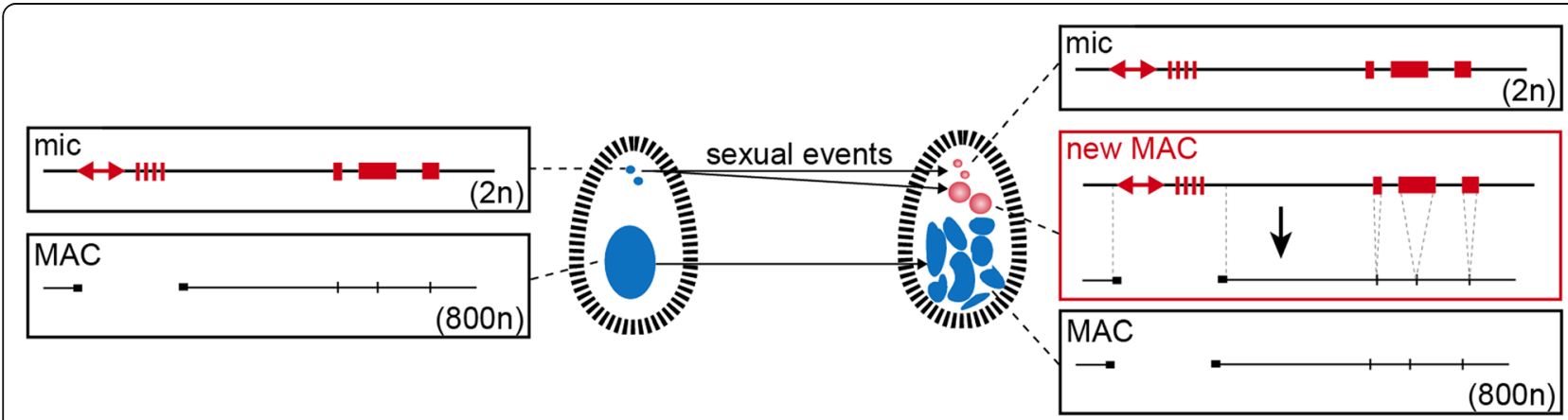

Fig. 1 Nuclear dimorphism and programmed DNA elimination in Paramecium tetraurelia. Left panel. Each cell contains two distinct types of nuclei: two diploid germline micronuclei (MIC, 2n) and one highly polyploid somatic macronucleus (MAC, 800n). Both nuclei develop from copies of the zygotic nucleus after fertilization. Right panel. Massive and reproducible elimination of germline DNA occurs during macronuclear development. Imprecise elimination of germline DNA containing repetitive sequences such as minisatellites (hatched rectangle) and transposable elements (double-headed arrow) is associated with the fragmentation of germline chromosomes into shorter macronuclear molecules healed by de novo telomere addition (black rectangles). In addition, 45,000 short, non-coding Internal Eliminated Sequences (IESs) (red rectangles) scattered throughout the germline genome are precisely excised from coding and intergenic sequences, restoring open reading frames and allowing gene expression 
disintegrates into about 30 small pieces that persist in the cytoplasm, while new MICs and MACs, destined for the progeny, develop from a copy of the diploid zygotic nucleus. Thus, new developing MACs coexist with the two MICs and about 30 small fragments of the maternal MAC (Fig. 2a). We used a published procedure to fractionate the nuclei of Pgm-depleted cells [3] (Fig. 2b). Briefly, nuclei from lysed cells were separated from contaminating organelles and cell debris on a sucrose cushion. The nuclear fraction, containing a mixture of different types of nuclei, was then submitted to flow cytometry (Additional file 1: Figure S1).

A fully developed MAC has a ploidy of 800n [10]. Therefore, new MACs at an advanced developmental stage emit a more intense DAPI (DNA staining) signal than the other nuclei present in the cell at the same stage (MICs and fragments of the maternal MAC). They are also considerably larger than the other nuclei, to accommodate this large amount of chromatin, and are spherical in shape (Fig. 2a). Taking advantage of these characteristics, we FACS-sorted new MACs $(\sim 15 \mu \mathrm{m})$ according to size (Forward-scattered light, FSC), granularity (Side-scattered light, SSC), pulse width and DAPI signal (Fig. 2c). Purity was measured by flow cell imaging before and after sorting. The developing MAC fraction, that represented $54 \%$ of the total nuclear sample before sorting, was enriched to $98 \%$ after sorting (Fig. 2d-e). Thus, the sorting procedure conferred considerable improvement over the pre-existing protocol.

To further validate the sorting procedure, we performed high throughput Illumina sequencing of DNA extracted from 266,000 sorted developing MACs ("sorted PGM DNA") (Additional file 2: Table S1). To identify the IESs in a sequencing sample, we used our previously published pipeline [11]. A total of 44,947 IESs was identified in the sorted PGM DNA, compared to 44,928 IESs in unsorted PGM DNA [3]. The fact that 97\% $(n=43,839)$ of the IESs identified in the sorted PGM DNA correspond to the same IESs identified in unsorted PGM DNA testifies to the reliability of our procedure. The $3 \%$ difference lies within the estimated error rate of the method $[3,11]$.

We then quantified the enrichment of our samples in un-rearranged sequences, by calculating a retention score for each of the 44,928 IES sequences present in the previously published $P$. tetraurelia IES reference set [3]. Retention score values range from 0 for no IES retention to 1 for complete IES retention, when the IES is retained in all sequenced copies of the genomic locus in question. As expected (Fig. 2f), retention score distribution in the rearranged MAC DNA control sample is close to 0 (mean 0.005), whereas a Gaussian distribution is observed for the unsorted non-rearranged PGM DNA, with a mean retention score of 0.69. Even if the Pgm endonuclease is required for all IES excision events, the mean retention score of this sample can never reach 1 , because the un-rearranged DNA from the developing new MACs is present in the unsorted sample alongside rearranged DNA from the fragments of the maternal MAC. By contrast, the sorted PGM DNA gave a Gaussian distribution with a mean retention score of 0.82 . This higher retention score, obtained from the same starting material, reflects greater enrichment in un-rearranged DNA, and thus in developing nuclei, providing further validation for the superiority of the sorting procedure over the existing protocol. In conclusion, this experiment establishes the proof of principle that nuclei can be sorted from a complex biological sample to high purity based on their size, shape and DNA content.

\section{Purification of MICs from vegetative cells}

We used a similar strategy to sort the small germline MICs from vegetative cells (Fig. 3 and Additional file 1: Figure S1). The available MIC isolation method, that relies on Percoll density gradient centrifugation [7], does not provide a MIC fraction sufficiently pure for exclusive MIC genome sequencing, owing to contamination from i) the MAC DNA (800n vs $2 \mathrm{n}$ in MIC), and ii) bacteria, on which Paramecium cells feed. MIC isolation has been achieved in other ciliates [12-14] but the same methods were not successful in Paramecium. We hypothesized that the contamination issues can be solved by the use of a specific fluorophore that is unambiguously and exclusively associated with the MICs. We previously generated transgenic Paramecium cells that constitutively express a MIClocalized version of the Green Fluorescent Protein (GFP) fused to centromeric histone H3 (CenH3a) [15]. Transgenic CENH3a-GFP cells have green fluorescent MICs, but neither the MAC nor the bacteria are GFP positive (Fig. 3a). We used the same fractionation scheme as the one previously published, with some improvements, to enrich for MICs [7] (Fig. 3b), and submitted the sample to flow cytometry. MICs were sorted based on the SSC, FSC, DAPI (DNA staining) and GFP signals (Fig. 3c-d). The procedure was optimized by flow cell imaging to define the population of interest and refine the sorting parameters (Additional file 1: Figure S1). We obtained 528,000 MICs from 3 million cells.

As previously, purity before and after sorting was measured by flow cell imaging. The MICs represented only $3 \%$ of the total sample before sorting and $97 \%$ after sorting (Fig. 3e-f). Thus, the sorting procedure is indispensable for effective MIC purification. We performed high throughput Illumina sequencing of the DNA extracted from sorted MICs (528,000 sorted MICs; $60 \mathrm{ng}$ ) and from the MIC-enriched sample before sorting. As expected, the bacterial DNA contamination greatly diminished after sorting $(8.2 \%$ of known contaminants before and $0.2 \%$ of 
a

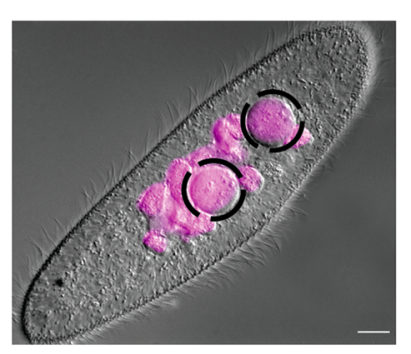

C
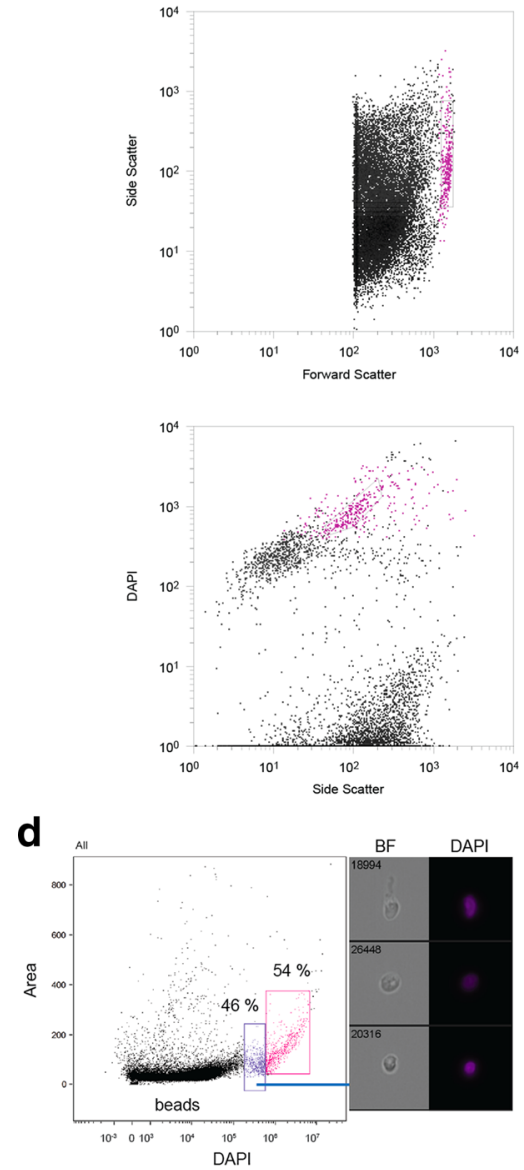
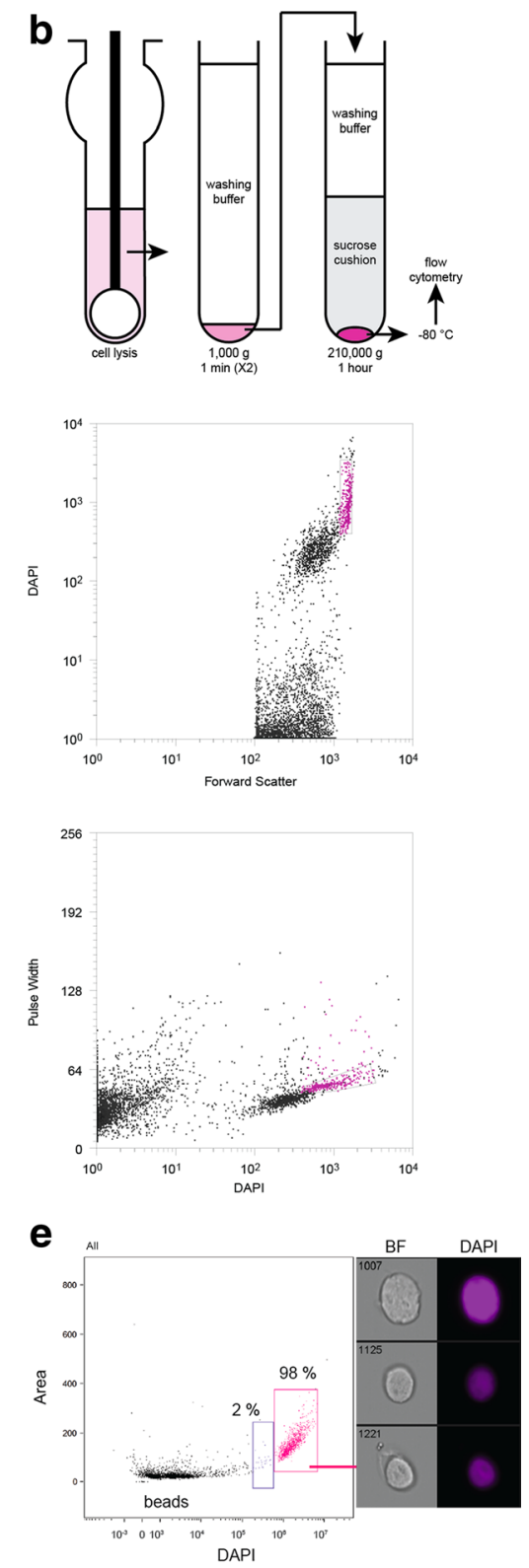

f

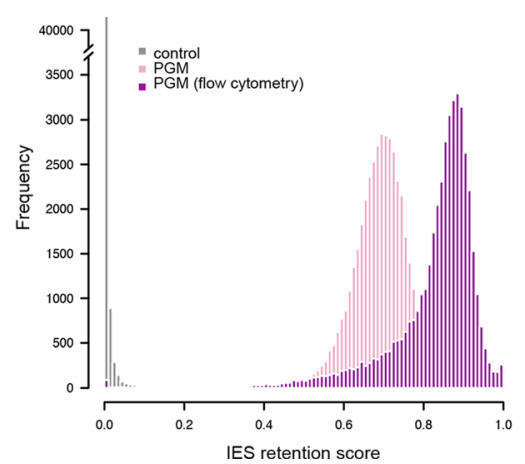

Fig. 2 (See legend on next page.) 


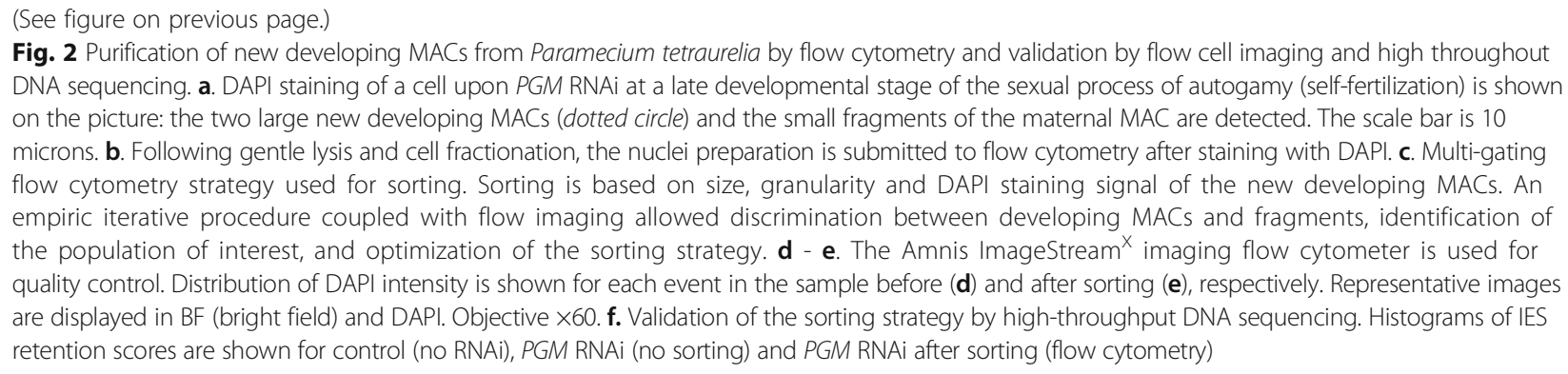

known contaminants after sorting) (Additional file 2: Table S1). We identified 44,851 IESs in the sorted sample, but only 5,192 IESs in the unsorted nuclear fraction. Calculation of mean IES retention scores indicated that enrichment in MIC-limited sequences increased from 0.04 in MIC DNA to 0.38 in sorted MIC DNA (Fig. 3g). The fact that $97 \%$ MIC purity only led to approximately $40 \%$ MIC DNA is explained by the much higher DNA content of the 3\% MAC-derived contaminating fraction. We conclude that flow cytometry sorting is necessary to directly sequence all IESs in unperturbed cells. The fact that $97 \%(n=43,741)$ of the IESs identified in the sorted MIC DNA correspond to the same IESs identified in the sorted PGM DNA confirms that the genome-wide set of IESs in PGM DNA reflects the complete set of MIC IESs (Additional file 1: Figure S2). These data demonstrate that the Pgm domesticated transposase is required for the excision of all IESs.

\section{A first glimpse of the germline genome reveals new transposable elements}

The sequence complexity of the MIC assembly is presented in Table 1 . Coverage by MAC reads was used to define MAC-destined as opposed to MIC-limited compartments. The $98 \mathrm{Mb}$ assembly consists of $74 \mathrm{Mb}$ ( $75 \%)$ of MAC-destined sequences and $24 \mathrm{Mb}(\sim 25 \%)$ of MIC-limited sequences, consistent with the size of the MAC reference genome assembly (72 $\mathrm{Mb},[6])$. It is important to realize that the MIC assembly we have obtained is highly fragmented (N50 $=37 \mathrm{~kb}$; half of the assembly is in contigs smaller than $37 \mathrm{~kb}$ ). The most fragmented part of the assembly is the MIC-limited compartment $(\mathrm{N} 50=13 \mathrm{~kb}$; half of the MIC-limited sequence is in contigs smaller than $13 \mathrm{~kb}$ ). With such an assembly, it is possible to annotate germline-limited elements such as IESs and transposable elements (TEs), but not to analyze long-range features such as chromosome structure. For that, additional information, e.g. from mate-pair libraries or third generation long read sequencing, is necessary to handle repeats and build scaffolds.

The MIC assembly consists of all of the contigs assembled using Velvet as launched by ParTIES [11] (Additional file 2: Table S2). The MIC-limited and the
MAC-destined parts of the assembly are defined as a function of MAC read depth, using the 3 MAC datasets described in (Additional file 2: Table S1). Any nucleotide with a MAC read depth $<20 \times$ is considered MIClimited, else the nucleotide is MAC-destined. N50 means that half an assembly is contained in contigs larger than the N50 value. The MIC-limited part of the assembly is thus much more fragmented than the MAC-destined part. The number of nucleotides covered by Internal Eliminated Sequences (IES), Transposable Elements (TE) and Tandem Repeats (TR) are given. MIC-limited sequences contain almost all IESs and TEs, $95.8 \%$ and $92 \%$ respectively. The majority (65\%) of TR are found in the MIC-limited sequences, however 35\%, reflecting WD40, TPR and other repeats, are found in the MAC-destined compartment.

The MIC contigs were used to identify TEs, starting from three previously identified Paramecium DNA transposons $[3,16]$ and a partial reverse transcriptase (RT) consensus (see Methods). tblastn searches using the DDE transposases or RT as queries identified a number of distinct elements, and potentially functional consensus sequences were reconstructed in most cases from the alignment of 10-20 copies (full range 4-48). The majority of TEs $(n=38)$ are Class I non-LTR retrotransposons, while 13 belong to the IS630-Tc1-mariner (ITm) super-family of Class II DNA transposons. The remaining consensus sequences are putative non-autonomous Class I SINE or solo-ORF1 elements. Characterization of the elements is provided (Additional file 3: Table S3 and Table S4). This analysis significantly augments knowledge of TE in the Paramecium germline and presents the first Paramecium Class I elements.

The non-LTR retrotransposons all have an ORF2 that contains both apurinic/apyrimidinic endonuclease (APE) and reverse transcriptase (RT) domains, like most known groups of non-LTR retrotransposons $[17,18]$. They fall in 5 groups, the first 3 of which also contain an upstream ORF1 (Fig. 4). A phylogeny was built using an alignment of the Paramecium RT domains with those of elements belonging to 11 previously characterized major clades [18] (Fig. 4, Additional file 1: Figure S3, Additional file 2: Table S5). The Paramecium retrotransposons, along with elements from 


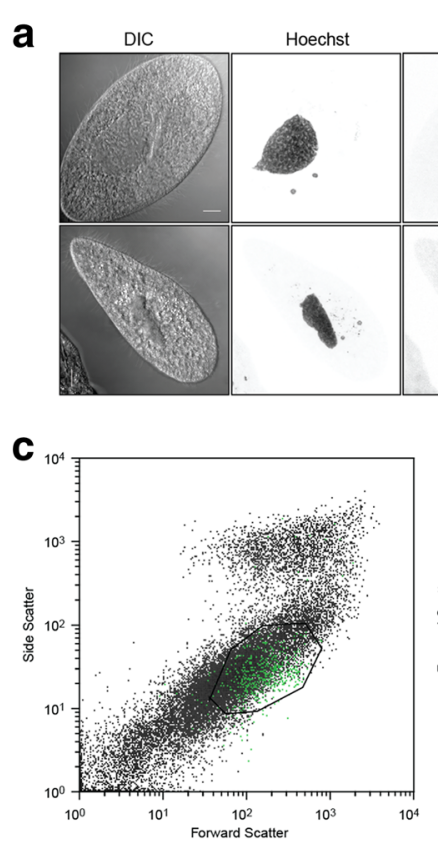

GFP
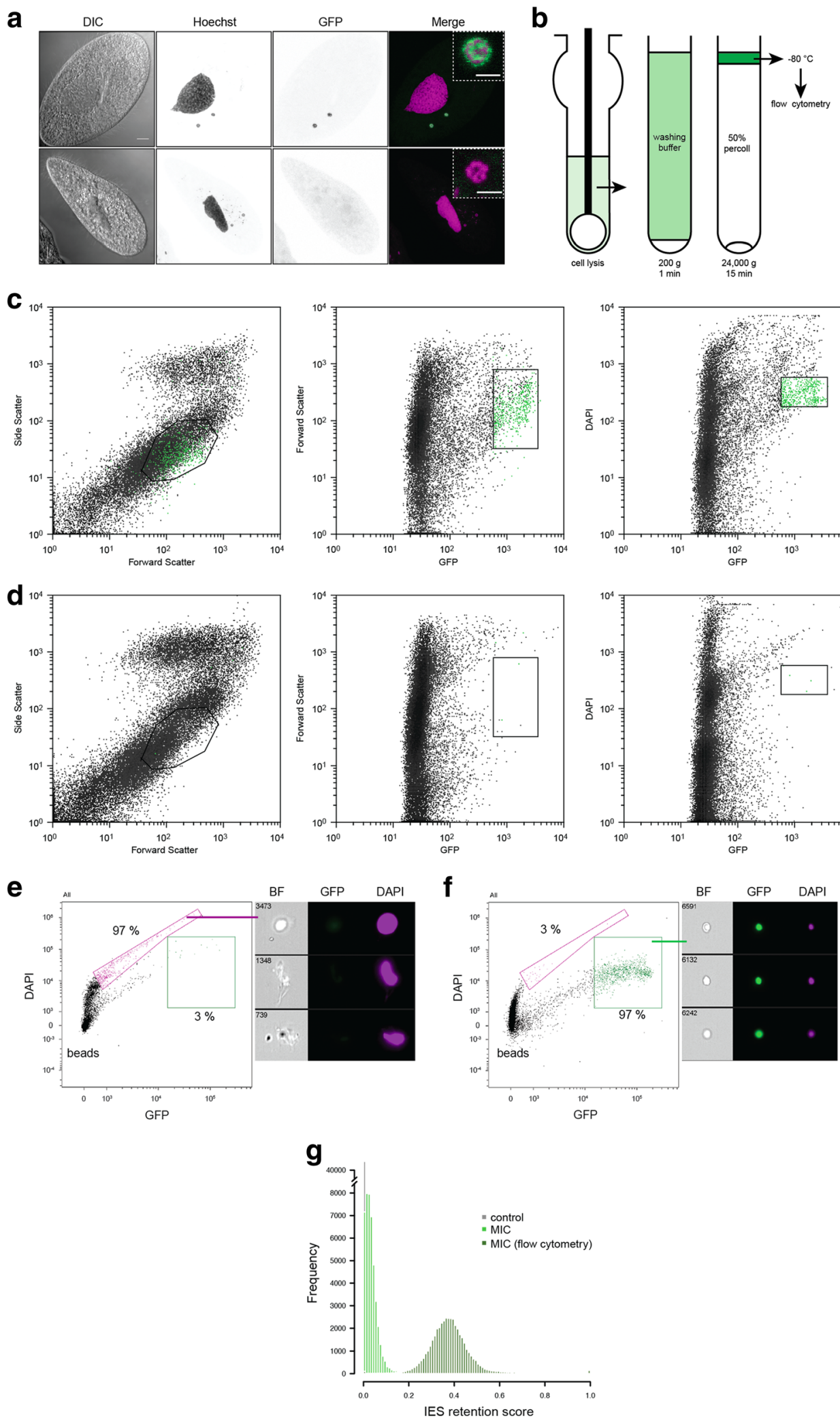

Fig. 3 (See legend on next page.) 
(See figure on previous page.)

Fig. 3 Purification of germline MICs from vegetative Paramecium tetraurelia by flow cytometry and validation by flow cell imaging and high throughout DNA sequencing. a. In CENH3a-GFP transgenic Paramecium vegetative cells (upper panels), but not in control cells (Iower panels), the MICS are GFP positive. Scale bar is 10 microns. Higher magnification: Scale bar is 3 microns. b. Fractionation scheme used to isolate the MIC-enriched fraction. $\mathbf{c}$ - $\mathbf{d}$. Multi-gating strategy used for sorting the MICs. Sorting is based on size, granularity and DAPI staining and GFP signals in c) CENH3a-GFP transgenic cells and $\mathbf{d}$ ) control cells. An empiric iterative procedure coupled with flow imaging allowed discrimination between MICs and DAPI containing contaminants, identification of the population of interest, and optimization of the sorting strategy. e - $\mathbf{f}$. The Amnis ImageStream ${ }^{X}$ imaging flow cytometer is used for quality control: sample before (e) and after sorting (f). g. Validation of the sorting strategy by high-throughput DNA sequencing. Histograms of IES retention scores are shown for control (no RNAi), MIC (no sorting) and MIC after sorting (flow cytometry)

the ciliate Tetrahymena thermophila [19], emerge as a distinct new clade in the tree, with good branch support. The consensus sequences of the first 3 groups, which contain an ORF1, suggest that ORF2 translation depends on +1 ribosomal frameshifting or translation re-initiation (Groups 1 and 2), or on translational read-through of the ORF1 stop codon (Group 3). Like other non-LTR retrotransposons [20,21], these elements contain short stretches of variable tri-, tetra-, or penta-nucleotide repeats at their 3' ends (Additional file 3: Table S3). Seven elements (solo ORF1s A-G) appear to contain only an ORF1, ending with a zinc finger similar to that found at the C-terminal end of ORF1 in Groups 1-3, and are likely mobilized in trans by proteins encoded by other elements; a (TAAA) $n$ repeat was found at the end of the element in 3 cases.

The 13 DNA transposons, all of the ITm superfamily [22], are unusual in that they contain multiple ORFs (Additional file 3: Table S4). In addition to the DDE ORF common to all ITm elements, an ORF2 of unknown function is found in all Paramecium transposons and shares detectable sequence similarity among all of them (Additional file 1: Figure S4). The largest Paramecium transposons contain 4 ORFs, ORF4 being a tyrosine recombinase, a property shared with TEC and TBE transposons from distantly related ciliates [23-25]. As seen in the Maximum Likelihood tree built using many ITm DDE domains [22, 26] (Fig. 5, Additional file 1: Figure S5, Additional file 2: Table S6), the composite Paramecium elements with a tyrosine recombinase group together, along with TEC1 and TEC2. A distance of 32 aa between the second and third residues of the DDE catalytic triad, characteristic of the 7 tyrosine-recombinase containing Paramecium ITm and 3 of the 6 simpler elements, is among the shortest ever reported for ITm.
RepeatMasker was used to identify copies of the TEs in the MIC contigs. Tandem Repeat Finder was used to identify putative satellite sequences (see Methods). As shown in Table 1, 96\% of the short unique copy IESs and $92 \%$ of the TE copies are in the MIC-limited compartment. However, about one third of tandem repeats were found in the MAC-destined compartment and include WD40, TPR and surface antigen repeats.

\section{MIC and PGM DNA are not equivalent}

To compare the sorted MIC DNA with the unrearranged DNA from PGM-silenced cells, used until now to represent germline DNA, we calculated the depth of coverage of the MIC assembly by the sorted MIC DNA and the sorted PGM DNA sequencing datasets. The calculation was performed for 90,017 non-overlapping 1-kb windows.

We visualized the comparison between the two datasets by creating dot plots of the depth for each window, and representing the density of the dots using heat map colors. To help interpret the comparison, we simulated PGM and MIC datasets, using enrichments in MIClimited sequences of 80 and $40 \%$ respectively (see Methods). As shown in Fig. 6a left plot, the simulated data present two clouds of points. The larger cloud, with the higher depth of coverage in both samples, corresponds to windows present in both the MIC and the MAC DNA. The smaller cloud, with lower depth of coverage in both samples, represents sequence windows present only in MIC DNA. The real data deviates from this unbiased profile (Fig. 6a, right). The larger clouds representing windows present in both MIC and MAC DNA are comparable (Additional file 1: Figure S6). Surprisingly, the smaller cloud is now vertically elongated, indicating that genome coverage in the PGM

Table 1 Characterization of MIC contigs

\begin{tabular}{llll}
\hline & MIC assembly & MAC-destined & MIC-limited \\
\hline Complexity & $98489268 \mathrm{bp}$ & $74212942 \mathrm{bp}(75.4 \%)$ & $24276326 \mathrm{bp}(24.6 \%)$ \\
N50 & $37.2 \mathrm{~Kb}$ & $46.9 \mathrm{~Kb}$ & $12.7 \mathrm{~Kb}$ \\
GC content & $27.40 \%$ & $27.97 \%$ & $25.66 \%$ \\
IES & $3517996 \mathrm{bp}$ & $147387 \mathrm{bp}(4.2 \%)$ & $3370609 \mathrm{bp}(95.8 \%)$ \\
Transposable Elements & $2973685 \mathrm{bp}$ & $237838 \mathrm{bp}(8 \%)$ & $2735847 \mathrm{bp}(92 \%)$ \\
Tandem Repeats & $1393130 \mathrm{bp}$ & $485112 \mathrm{bp}(34.8 \%)$ & $908018 \mathrm{bp}(65.2 \%)$ \\
\hline
\end{tabular}




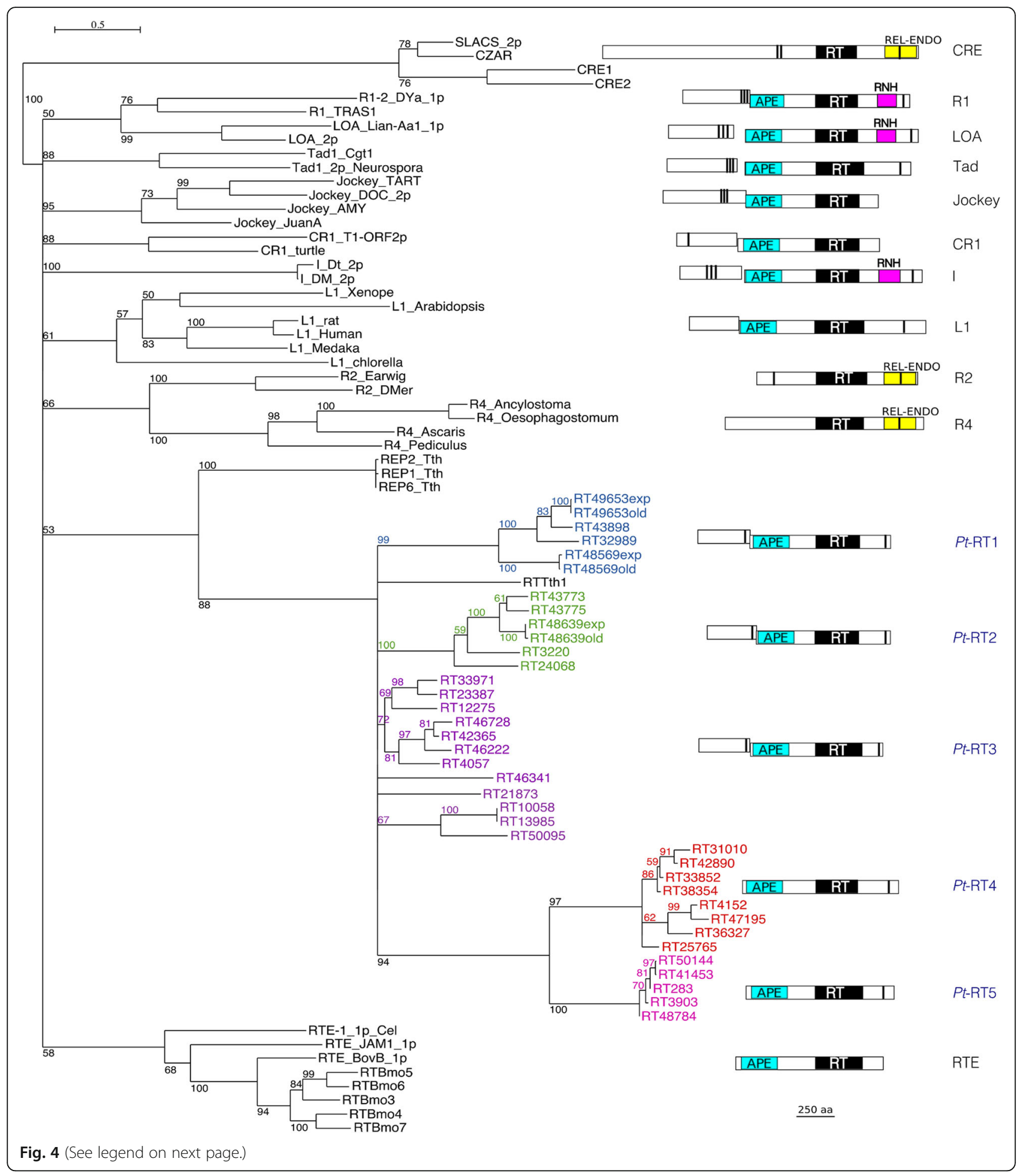




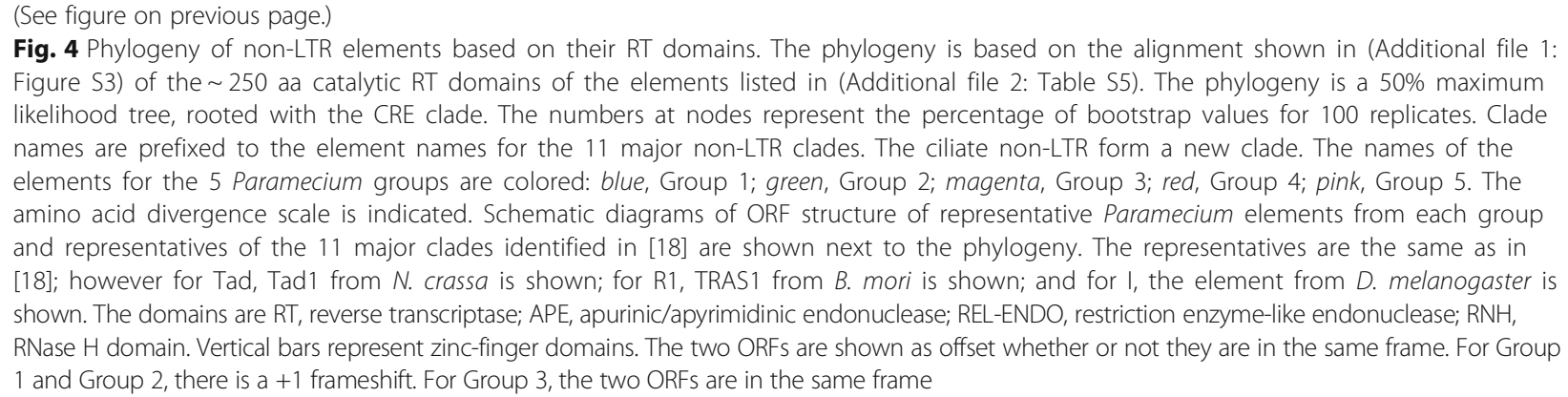

DNA is variable and mostly less covered than expected (depth between 0 and 7). Both PGM samples behave in the same way. The same windows are found to be under-represented in both PGM and unsorted PGM samples (Additional file 1: Figure S6).

To refine this observation and determine which sequences are missing from the PGM DNA, we used the uniquely-mapped read counts in the 1-kb non-overlapping windows to identify differentially covered windows, in the same way as RNA-Seq counts for genes are used to identify differentially expressed genes (see Methods). The statistical software package we used takes into account the small number of independent samples ( 2 or 3 biological replicates for most samples, Additional file 2: Table S1).

We looked for windows less covered by MAC or PGM reads with respect to MIC reads (Additional file 1: Figure S7). This allowed us to define three genomic compartments (Fig. 6b, Table 2): $80 \%$ of the MIC genome non-overlapping windows are not differentially covered and represent the part of the germline genome that is collinear with MAC chromosomes ("MAC-destined"). The remaining $20 \%$ of the windows was significantly less covered by MAC than by MIC reads, corresponding to the germline-limited part of the genome ("MIC-limited"). As anticipated by the previous analysis of read depth, 3\% of the windows not covered by MAC reads are not wellcovered by the PGM reads. We thus subdivided the MIC-limited compartment into "MIC PGM" and "MIConly" sub-compartments (Fig. 6b). Figure 6c shows barplots of the normalized read counts of the windows for each of the samples, for the "MIC PGM" and "MIConly" sub-compartments. As expected, the two subcompartments are not covered by MAC reads and are well-covered by MIC reads. Interestingly, the "MIConly" sub-compartment, which is poorly covered by PGM reads, is well-covered by DCL2/3 and EZL1 reads (Additional file 1: Figure S6). These two factors are required for developmental DNA elimination and act respectively in small RNA and histone post-translational modification pathways upstream of the introduction of DNA double-strand breaks by the Pgm endonuclease [27].
Columns from left to right: "MAC-destined" is the genomic compartment covered by MIC, MAC and PGM reads (i.e. windows with no differential coverage according to the DESeq2 analysis, see Methods); "MIC PGM" is the sub-compartment covered by MIC and PGM reads; "MIC only" is the sub-compartment covered only by MIC reads. These compartments are represented schematically in Fig. 6b. The IES reference set was mapped to the MIC assembly and then the IESs were assigned to a window. The total complexity of tandem repeats (micro- and mini-satellite) was calculated using Tandem Repeats Finder. Low complexity sequences identified by Repeat Masker include stretches of polypurine or poly-pyrimidine and regions of high AT (>87\%) or high GC (>89\%) content. Repeat Masker was also used to find TE copies, using the TE consensus library reported in this study (See Methods, Additional file 4: Text S1 and Text S2. The difference between the "MIC PGM" and the "MIC only" sub-compartments was judged highly significant for Tandem repeats and for TE ( $p$-value: $9.88 \mathrm{e}-324$ and $9.45 \mathrm{e}-105$, respectively). The MIC only sub-compartment, representing germlinelimited sequences not present in either of the PGM samples, is thus enriched in satellites and depleted in TEs.

Different sequence characteristics were calculated for the three genomic sub-compartments (Table 2). GC content and low complexity content did not vary across sub-compartments. Approximately $99 \%$ of the IES reference set could be mapped to the MIC assembly. Since 90\% of IESs are shorter than 100 bp (median IES size $51 \mathrm{nt}$ ) it is not surprising that nearly all IES-containing $1-\mathrm{kb}$ windows are covered by MAC reads and are thus found in the "MAC-destined" compartment.

The TE consensus library was used to find TE copies in the 3 genomic sub-compartments (Table 2). The important difference between the $16 \mathrm{Mb}$ "MIC PGM" and the $3 \mathrm{Mb}$ "MIC-only" sub-compartments is that the latter is significantly depleted in TE copies and enriched in tandem repeats i.e. micro- and mini-satellite (Table 2).

We can suggest two possible, non-exclusive explanations for why $3 \mathrm{Mb}$ of sequence complexity present in 


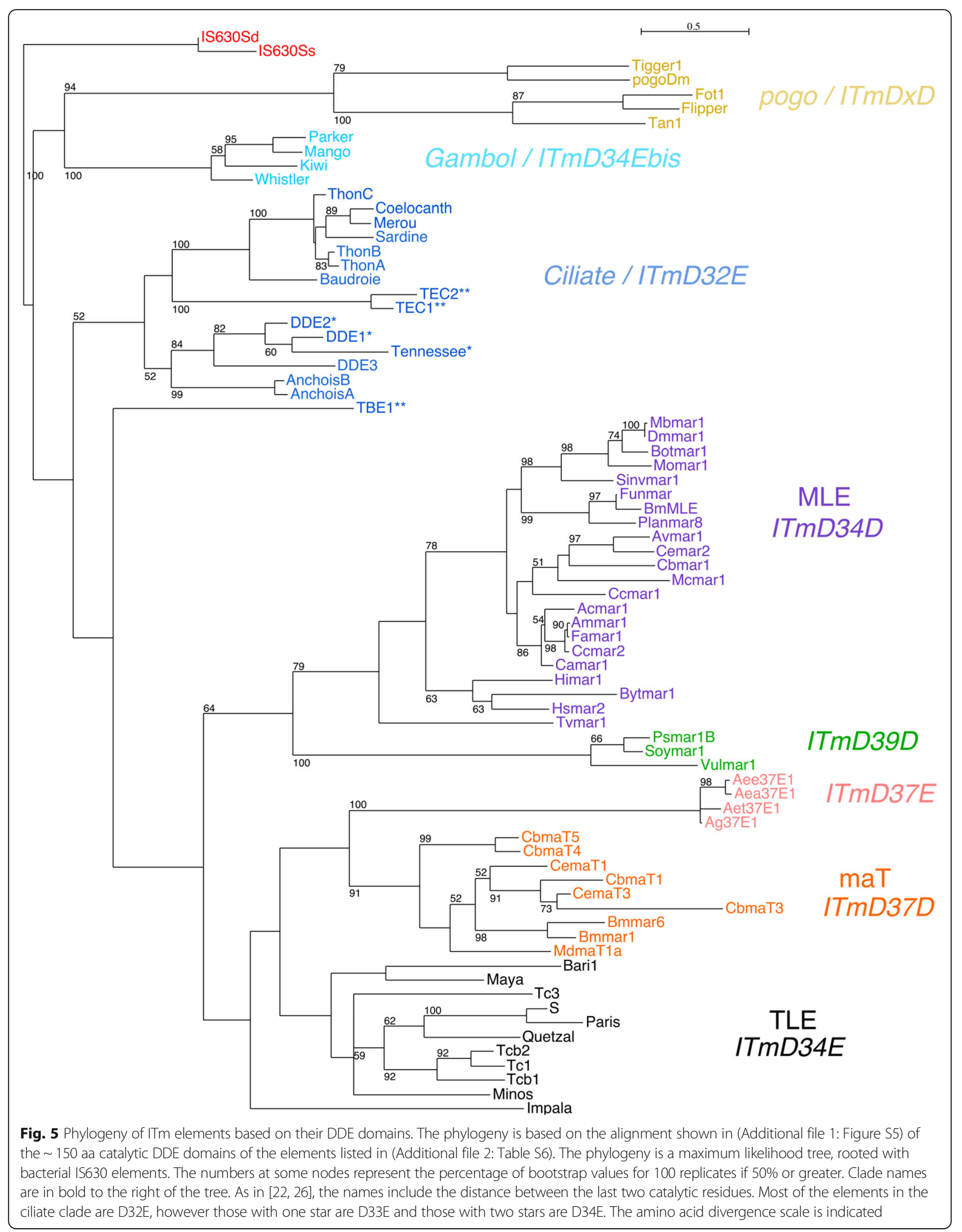



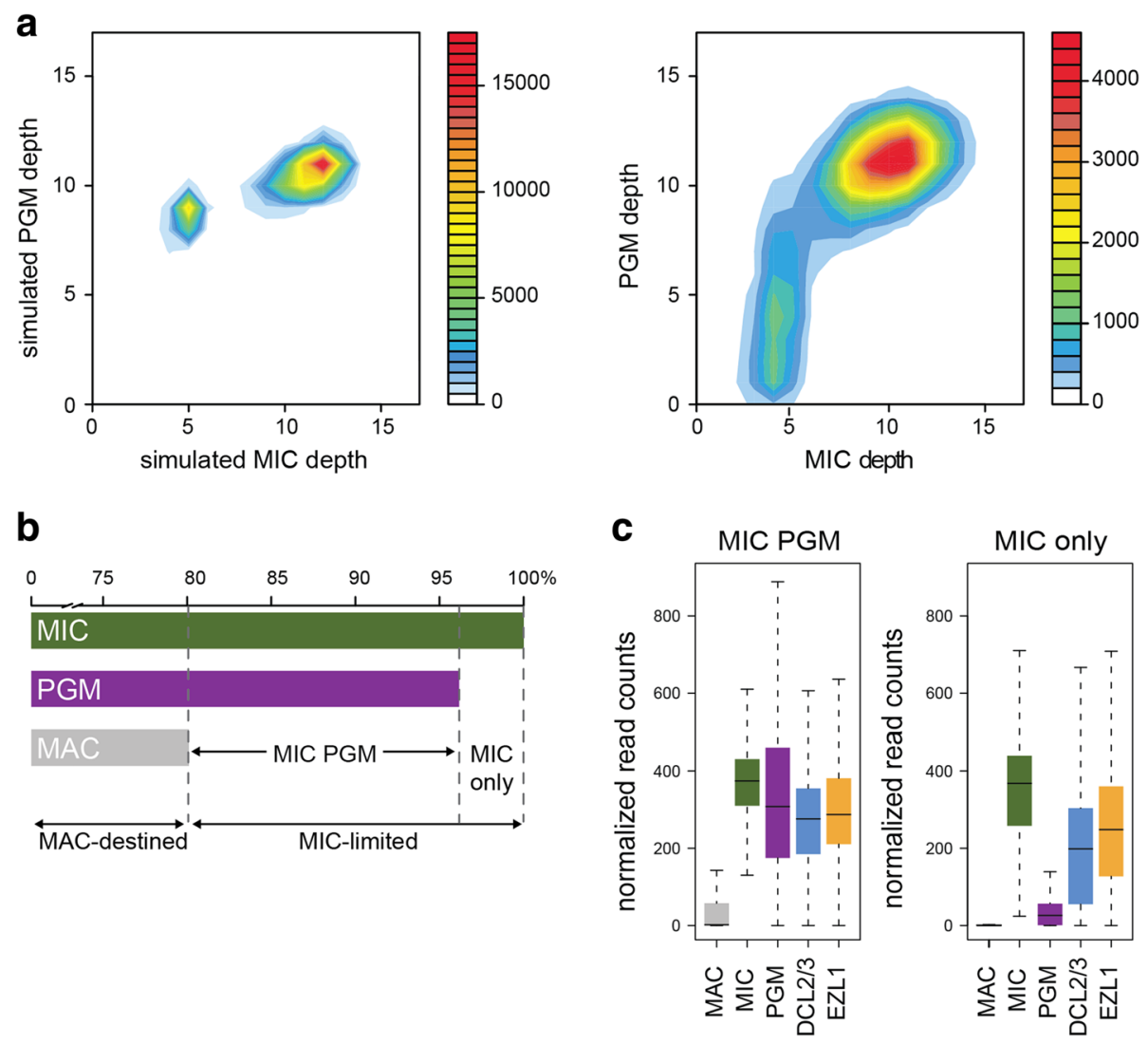

Fig. 6 Coverage of the MIC assembly by different sequencing samples. a. Global comparison of the sequences in simulated (left) and real (right) PGM and MIC sequencing samples. Depth is calculated by mapping reads to the MIC assembly, and counting the reads in 1-kb non-overlapping windows. The graph shows the density of windows as a heat map color, for each combination of MIC and PGM normalized depth values. $\mathbf{b}$. Representation of the genomic compartments identified by analysis of differential read coverage of the MIC assembly (cf. Methods, DESeq2 analysis and Table 2). The horizontal bars show the percentage of the MIC assembly covered by each sequencing sample, defining three genomic sub-compartments. "MAC-destined" is the genomic compartment covered by MIC, MAC and PGM reads, i.e. windows with no differential coverage according to the DESeq2 analysis; "MIC PGM" is the compartment covered by MIC and PGM reads; "MIC-only" is the compartment covered only by MIC reads. c. Barplots of the normalized DESeq2 read counts, across all windows and all samples (Additional file 2: Table S1) for the "MIC PGM" compartment (left) and the "MIC-only" compartment (right)

Table 2 Characterization of different sub-compartments of the MIC assembly

\begin{tabular}{llll}
\hline & MAC-destined & MIC PGM & MIC only \\
\hline Complexity & $76130194 \mathrm{bp}$ & $15983936 \mathrm{bp}$ & $2905995 \mathrm{bp}$ \\
Genome proportion & $80.12 \%$ & $16.82 \%$ & $3.06 \%$ \\
Longest contiguous region & $286000 \mathrm{bp}$ & $58000 \mathrm{bp}$ & $79000 \mathrm{bp}$ \\
GC content & $27.58 \%$ & $26.29 \%$ & $27.47 \%$ \\
IES & $97.70 \%$ & $2.26 \%$ & $0.04 \%$ \\
Low complexity & $5.40 \%$ & $5.50 \%$ & $5.43 \%$ \\
Tandem repeats & $0.83 \%$ & $1.39 \%$ & $5.95 \%$ \\
TE & $1.33 \%(0.97 \mathrm{Mb})$ & $21.47 \%(3.43 \mathrm{Mb})$ & $7.35 \%(0.23 \mathrm{Mb})$ \\
not TE & $98.67 \%(75.16 \mathrm{Mb})$ & $78.53 \%(12.56 \mathrm{Mb})$ & $92.65 \%(2.68 \mathrm{Mb})$ \\
TIR & $0.45 \%(0.33 \mathrm{Mb})$ & $4.96 \%(0.79 \mathrm{Mb})$ & $0.53 \%(0.02 \mathrm{Mb})$ \\
LINE & $0.84 \%(0.62 \mathrm{Mb})$ & $15.98 \%(2.56 \mathrm{Mb})$ & $6.57 \%(0.2 \mathrm{Mb})$ \\
SINE & $0.04 \%(0.03 \mathrm{Mb})$ & $0.53 \%(0.08 \mathrm{Mb})$ & $0.25 \%(0.01 \mathrm{Mb})$ \\
\hline
\end{tabular}


the MIC DNA are absent from the PGM DNA: i) the Pgm domesticated transposase is not needed for the elimination of some MIC-limited sequences or ii) PGM RNAi is released at the end of development and this release is sufficient for elimination of some MIC-limited sequences. Consistent with the latter explanation, depletion of other factors involved in programmed DNA elimination, and whose function is likely upstream of Pgm, did not lead to underrepresentation of MIC-limited sequences (Fig. 6c and Additional file 1: Figure S6). Whatever the reason of the under-representation of MIC-limited sequences upon PGM RNAi, it indicates that PGM DNA, used up until now as a proxy for MIC DNA, does not provide a faithful representation of the MIC genome.

\section{Conclusions}

We report the development of an efficient flow cytometry-based method to sort nuclei in P. tetraurelia. This method represents a major breakthrough over previously published methods [3, 7], in that it provides (i) improved reliability; (ii) high purity; and (iii) quality control evaluated by flow cell imaging and high throughput sequencing. Our work also provides a clear demonstration that flow cell imaging is a powerful means to detect the population of interest and help refine sorting parameters.

We expect that cytometry-based purification of subpopulations of macronuclei during development may allow kinetic studies of the DNA elimination and endoreplication processes. We have shown that our procedure allows high throughput Illumina sequencing of the $P$. tetraurelia germline genome, paving the way for sequencing the germline genome of other $P$. aurelia sibling species for future comparative genomic studies.

So far, only a few studies have made use of flow cytometry to sort nuclei [28-40], mostly in plants and neurons. Our work highlights the unique potential of flow cytometry to analyze and sort heterogeneous populations of nuclei. It demonstrates that flow cytometry and sorting provide a powerful way to purify minority subpopulations of nuclei, provided that specific nuclear characteristics or a specific fluorophore can be unambiguously and exclusively associated with the subpopulation of interest.

The contigs assembled from the sorted MIC DNA have allowed discovery of 61 germline TEs. The majority are Class I non-LTR retro-transposons (LINE elements), never before characterized in Paramecium. This library of manually curated TE consensus sequences constitutes a precious resource for future automated approaches to TE identification and classification in the germline genomes of Paramecium species, especially given the relatively large phylogenetic distances to related elements from other taxa.

\section{Methods}

\section{Cells and cultivation}

All experiments were carried out with the entirely homozygous strain 51 of Paramecium tetraurelia. Cells were grown in a wheat grass powder (WGP, Pines International, USA) infusion medium bacterized the day before use with Klebsiella pneumoniae, unless otherwise stated, and supplemented with $0.8 \mathrm{mg} / \mathrm{L}$ of $\beta$-sitosterol (Merck). Cultivation and autogamy were carried out at $27^{\circ} \mathrm{C}$.

\section{Developing MAC purification}

We used the feeding method described in [41] to silence the PGM gene. Escherichia coli HT115 [42] harboring plasmid L4440 [43], with the 567-bp HindIII-NcoI fragment of the PGM gene inserted between two convergent T7 promoters [9], was induced for the production of PGM dsRNA in WGP1X medium containing $100 \mu \mathrm{g} / \mathrm{mL}$ ampicillin by overnight growth at $37{ }^{\circ} \mathrm{C}$ with shaking. The next day, the culture was diluted into the same medium to OD600 $=0.04$. IPTG (Euromedex) was added at a final concentration of $0.4 \mathrm{mM}$ to induce dsRNA synthesis. After $4 \mathrm{~h}$ of induction at $37{ }^{\circ} \mathrm{C}$ with shaking, the medium was cooled down to $27{ }^{\circ} \mathrm{C}$, and supplemented with $0.8 \mathrm{mg} / \mathrm{L}$ of $\beta$-sitosterol just before use.

$P$. tetraurelia cells were first grown in standard $K$. pneumoniae medium for 20-30 vegetative fissions then washed twice in silencing medium. Cells were allowed to grow for 8 to 10 vegetative fissions in a final volume of $3 \mathrm{~L}$ of silencing medium (freshly induced medium was added the second day) then starved to trigger autogamy. Progression of autogamy was monitored by Hoechst staining (Sigma). At day 4 of starvation, 30 autogamous cells were picked and transferred individually to $200 \mu \mathrm{L}$ of $K$. pneumoniae medium to monitor the viability of sexual progeny and evaluate the efficiency of PGM silencing. As expected, $P G M$ RNAi led to high rates of lethality in the sexual progeny.

At day 4 of starvation, cells were $100 \%$ autogamous with about $90 \%$ of cells displaying two large developing MACs. Purification of developing new MACs was performed using the protocol described in [3] with minor modifications. Cultures were filtered on 8 layers of sterile gauze. Cells were centrifuged at $600 \mathrm{~g}$ for $1 \mathrm{~min}$ in an oil-testing centrifuge (Sigma 6-16, rotor 13116) then washed in $100 \mathrm{~mL}$ of Tris- $\mathrm{HCl} 10 \mathrm{mM} \mathrm{pH} \mathrm{7.4} \mathrm{and} \mathrm{cen-}$ trifuged again to obtain a compact pellet $(\sim 1 \mathrm{~mL})$. After centrifugation, the cell pellet was resuspended in 2 volumes of lysis buffer $(\sim 2 \mathrm{~mL})(0.25 \mathrm{M}$ sucrose; $10 \mathrm{mM}$ $\mathrm{MgCl} 2 ; 10 \mathrm{mM}$ Tris $\mathrm{pH}$ 6.8; 0.2\% NP40) and kept on ice for $5 \mathrm{~min}$. All steps were performed at $4{ }^{\circ} \mathrm{C}$. Cells were then lysed with a Dounce homogenizer until approximately $90 \%$ of the cells were broken as observed under a microscope $(\times 20)$. Developing MACs were collected by centrifugation at $1,000 \mathrm{~g}$ for $1 \mathrm{~min}$. The pellet that 
contained the developing MACs was washed twice with 9 volumes $(\sim 9 \mathrm{~mL})$ of washing buffer. The pellet was then resuspended in $2 \mathrm{~mL}$ sucrose solution $(2.1 \mathrm{M}$ sucrose; $10 \mathrm{mM} \mathrm{MgCl}$; $10 \mathrm{mM}$ Tris $\mathrm{pH}$ 7.4) and loaded on top of a $3 \mathrm{~mL}$ sucrose solution layer in an Ultra-clear centrifuge tube (Beckman Coulter 344059). After gentle addition of washing buffer to fill the tubes, the samples were centrifuged at $210,000 \mathrm{~g}$ for $1 \mathrm{~h}$, in a SW41ti swinging rotor (Optima L-80 XP ultracentrifuge, Beckman Coulter). After centrifugation, the sucrose solution was carefully removed. The pellet was gently rinsed with washing buffer, before resuspension into $\sim 3 \mathrm{~mL}$ of washing buffer containing glycerol ( $13 \%$ final concentration). The samples were aliquoted and frozen at $-80{ }^{\circ} \mathrm{C}$.

\section{Micronuclei purification}

Transgenic Paramecium cells expressing a micronuclear (MIC)-localized version of the Green Fluorescent Protein (GFP) were obtained by microinjection of the vegetative macronucleus with the CenH3a-GFP plasmid, described in [15], in which the centromeric histone variant (CenH3a) gene fused to GFP is expressed under the control of the constitutive promoter of the elongation factor $\mathrm{Tu}$. In the transformed clones, GFP was exclusively found in the MICs. Transformed clones were selected for their GFP signal/noise ratio. Transgene quantification indicated a copy number close to the endogenous CenH3a gene level (transgene/endogenous gene $\sim 0.6$ to 1 ). Viability of the sexual progeny after autogamy of the transformed clones was systematically monitored to make sure that the presence of the transgene did not impair the functionality of the MICs.

Transformed and non-injected cells were grown in standard $K$. pneumoniae medium in a final volume of $3 \mathrm{~L}$ at a cell density of 1,000 to 1,500 cells $/ \mathrm{mL}$. The vegetative state of the cells was assessed by nuclear staining with a $33: 1(\mathrm{vol} / \mathrm{vol}) \mathrm{mix}$ of carmine red $(0.5 \%$ in $45 \%$ acetic acid) and fast green (1\% in ethanol). Detection of GFP signal in the MICs was monitored in the transformed cells. Cultures were filtered on 8 layers of sterile gauze. Cells were centrifuged at $600 \mathrm{~g}$ for $1 \mathrm{~min}$ in an oil-testing centrifuge (Sigma 6-16, rotor 13116) then washed in $100 \mathrm{~mL}$ of Tris-HCl $10 \mathrm{mM} \mathrm{pH} 7.4$ and centrifuged again to obtain a compact pellet.

We used the same fractionation scheme as the one previously published to enrich in MICs [7] with some improvements. After centrifugation, the cell pellet was resuspended in 2 volumes of lysis buffer $(0.25 \mathrm{M}$ sucrose; $10 \mathrm{mM} \mathrm{MgCl} 2 ; 10 \mathrm{mM}$ Tris pH 6.8; 0.2\% NP40) and kept on ice for $5 \mathrm{~min}$. All steps were performed at $4{ }^{\circ} \mathrm{C}$. Cells were then lysed with a Dounce homogenizer until approximately $90 \%$ of the cells were broken as observed under a microscope $(\times 20)$. Three volumes of washing buffer $(0.25 \mathrm{M}$ sucrose; $10 \mathrm{mM}$ Tris $\mathrm{pH}$ 7.4;
$5 \mathrm{mM} \mathrm{MgCl} 2 ; 15 \mathrm{mM} \mathrm{NaCl} ; 60 \mathrm{mM} \mathrm{KCl} ; 0.5 \mathrm{mM}$ EGTA) were added. The sample was dispatched into $2 \mathrm{~mL}$ Eppendorf tubes and mixed by inversion 5 times then centrifuged at $200 \mathrm{~g}$ for $1 \mathrm{~min}$. The supernatant that contained most MICs was recovered and presence of the MICs was verified under a microscope. The supernatant was then transferred into Ultra-clear centrifuge tubes (Beckman Coulter 344059, $2 \mathrm{~mL}$ per tube), and $10 \mathrm{~mL}$ of $50 \%$ Percoll solution $(50 \%$ Percoll $\mathrm{pH} 7.5$; $0.25 \mathrm{M}$ Sucrose; $10 \mathrm{mM} \mathrm{MgCl} 2$ ) were added drop by drop with gentle agitation. The supernatant and the Percoll solution were gently mixed by pipetting and centrifuged at 24,000 g for $15 \mathrm{~min}$ in a SW41Ti swinging rotor (Optima L-80 XP ultracentrifuge, Beckman Coulter). During centrifugation, the Percoll gradient is formed and MICs accumulated at the top of the gradient and MACs at the bottom. After centrifugation, MICs were carefully recovered in a white-to-brown powderous band with a $200 \mu \mathrm{L}$ Pipetman into a $1.5 \mathrm{~mL}$ Eppendorf tube. The MIC-enriched sample was gently mixed then diluted 1/1/1 with washing buffer and glycerol 40\% (13\% glycerol final concentration). Usually several hundred MICs per microliter could be counted under a microscope. The samples were aliquoted and frozen at $-80{ }^{\circ} \mathrm{C}$ for further flow cytometry analysis and sorting.

\section{Flow cytometry}

Samples of MICs and developing MACs were thawed on ice, diluted $1 / 5$ to $1 / 10$ in washing buffer and stained with DAPI (3 $\mu \mathrm{M}$ final, Invitrogen \#D3571). All steps were performed at $4{ }^{\circ} \mathrm{C}$. The samples were filtered (30 $\mu \mathrm{m}$ Sysmex filters, 04-004-2326) and sorted on an Influx 500 cell sorter (BD Biosciences) with a $488 \mathrm{~nm}$ laser for scatter measurements (Forward Scatter, or FCS, and Side Scatter, or SSC) and GFP excitation, and a $355 \mathrm{~nm}$ laser for DAPI excitation. GFP and DAPI staining signals were collected using a $528-38 \mathrm{~nm}$ band pass filter and a $460-50 \mathrm{~nm}$ band pass filter, respectively. Phosphate Buffered Saline (Isoflow TM Sheath Fluid, Beckman Coulter) was used as sheath and run at a constant pressure of 15 PSI. Frequency of drop formation was $27 \mathrm{kHz}$. The instrument used a $100 \mu \mathrm{m}$ nozzle. For the MIC samples, a threshold on the GFP signal was optimized to increase collecting speed (2500 events per second). For developing MACs, an important threshold on FCS was optimized to not consider the crystals present in the sample and increase collecting speed. Paramecium cells contain crystals composed of guanine, xanthine and hypoxanthine [44], which are pelleted together with developing MACs during the purification procedure and can represent an important part of the elements detected by the instrument. Since they do not contain DNA, hiding crystals allowed a faster collecting speed without increasing DNA contamination. Sorting rates typically ranged from 
10,000 to 100,000 MICs per hour depending on the preparation. Data were collected using Spigot software. Micronuclei were sorted based on their SSC, FSC, GFP and DAPI signals. Events in GFP and DAPI gates were backgated onto FSC vs SSC to optimize the sorting. Developing MACs were sorted based on their SSC, FSC, DAPI, and time-of-flight (pulse width) signals. Events with high DPAI signal were backgated onto FSC vs SSC to optimize the sorting. Nuclei were recovered in washing buffer into a $1.5 \mathrm{~mL}$ Eppendorf tube.

\section{Flow cell imaging}

Purity of the sorted samples was evaluated by flow cell imaging. Samples before and after sorting were imaged on a 2 camera, 12 channel ImageStream ${ }^{\mathrm{X}}$ (Amnis/ MerckMillipore) imaging flow cytometer with a $60 \times$ magnification, using 405, 488, and $785 \mathrm{~nm}$ lasers, at respectively 125,100 , and $0.05 \mathrm{~mW}$. Phosphate Buffered Saline (137 mM NaCl; $2.7 \mathrm{mM} \mathrm{KCl} 6.7 \mathrm{mM} \mathrm{Na} 2 \mathrm{HPO} 4$; $1.5 \mathrm{mM} \mathrm{KH} 2 \mathrm{PO} 4)$ was used as sheath. Acquisitions were performed using Inspire software. Brightfield was collected in channel 1 and 9, SSC in channel $6(745-800 \mathrm{~nm}$ bandwidth), GFP in channel 2 (480-560 nm bandwidth), and DAPI in channel 7 (430-505 nm bandwidth). At least 5,000 elements were analyzed for each sample (before and after sorting) in order to detect enough MICs, given the rarity of MICs in the sample $(\sim 0.2-3 \%$ of all events detected by the Influx cell sorter before sorting). Cell classifiers were set for channel 1 area lower limit of 10 to allow the instrument to focus despite low concentration of the sample after sorting. Beads were excluded from the analysis based on their low DAPI and GFP fluorescence signals. Analysis was performed using the IDEAS software.

\section{Genomic DNA extraction and sequencing}

After sorting, MICs and developing MACs were treated with 3 volumes of proteinase $\mathrm{K}$ solution (0.5 M EDTA $\mathrm{pH}$ 9; $1 \%$ N-lauroylsarcosine; $1 \% \mathrm{SDS} ; 1 \mathrm{mg} / \mathrm{mL}$ proteinase $\mathrm{K})$ at $55{ }^{\circ} \mathrm{C}$ overnight. Genomic DNA was extracted with the addition of one volume of Tris-HCl-phenol $\mathrm{pH} 8$ with gentle agitation at room temperature for $1 \mathrm{~h}$ (no vortex). After centrifugation at $300 \mathrm{~g}$ for $15 \mathrm{~min}$, the aqueous phase was recovered, dialyzed twice against TE (10 mM Tris-HCl; 1 mM EDTA, pH 8) 25\% ethanol for $2 \mathrm{~h}$, against TE overnight, then against Tris $1 \mathrm{mM} \mathrm{pH} 8$ for $2 \mathrm{~h}$. DNA was concentrated with a Concentrator plus (Eppendorf) down to 50 to $100 \mu \mathrm{L}$. DNA concentration was quantified using QuBit High sensibility kit (Invitrogen) and stored at $4{ }^{\circ} \mathrm{C}$. DNA was then sequenced by a paired-end strategy using Illumina Hi-Seq next-generation sequencer (Additional file 2: Table S1). DNA-seq datasets have been deposited at the NCBI short read archive (SRA)
(Accession numbers: SAMN05323659; SAMN05323660; SAMN05323661).

\section{Transposable element annotation}

Putative LINE elements were discovered as follows. Reverse transcriptase coding domains were identified from a small cluster of homologous sequences retained in the MAC genome, after building a consensus from their alignment. These partial peptide sequences were then used to search the MIC contigs (tblastn using default parameters, with no low complexity filter). The matches were culled and used to extend the consensus protein sequences. Then blastn searches (default parameters, no low complexity filter) were used against the MIC contigs to find more copies. The procedure was used recursively to extend and find as many copies as possible. Copies were aligned with MUSCLE [45] and adjusted manually, with a requirement of potentially functional ORF1 and ORF2 sequences. Finally, the best adjusted consensus sequences were used to search for other related elements by a tblastn search for long, poorly scoring matches which might be recent copies of a different element. In this way, 5 distinct groups of LINE elements were found. A similar procedure was used to annotate Class II DNA transposons, starting from published sequences for the $P$. primaurelia Tennessee element ORFs [16] and the P. tetraurelia Sardine and Anchois element ORFs [3]. Finally, some sequences inserted in other elements were found to be present in multiple copies in the MIC assembly but yielded consensus sequences with no protein-coding potential; these sequences were annotated as putative SINE elements. Fasta files with the nucleotide and putative peptide sequences are provided (Additional file 4: Text S1-S2), (Additional file 3: Tables S3-S4).

\section{Phylogenetic tree reconstruction}

Non-LTR Class I retrotransposon ORF2 (pol) protein sequences representative of different clades [18] and IS630-Tc1-mariner (ITm) superfamily transposase protein sequences $[22,26]$ were recovered from GenBank or RepBase (Additional file 2: Tables S5-S6). Corresponding Paramecium consensus sequences were added to each set of proteins. The proteins were aligned using MSAProbs [46]. The alignments were trimmed manually to correspond to the RT and DDE catalytic domains, respectively (Additional file 1: Figures S3 and S5) and used for phylogenetic tree reconstruction by Maximum Likelihood $[47,48]$, with PhyML version 3.1 (PhyML -d aa -m LG -v 0.0 -c 4 -a E -f M -no_memory_check -i < phylip_alignment_file $>-\mathrm{b}$ 100). The non-LTR retrotransposon RT tree was collapsed if branch support (determined using 100 bootstrap replicates) was less than 50\%, using TreeGraph2 [49]. Seaview [50] was used 
for preliminary tree-building, to convert alignment formats and to visualize, re-root, swap branches and prepare figures of the trees.

\section{Bioinformatic analyses IES retention}

IES retention scores were calculated with ParTIES v1.0 [11] (MIRET module, -max_mismatch 1 -score -method Boundaries) using the $P$. tetraurelia IES reference set [3] and two reference genome assemblies available from ParameciumDB (http://paramecium.cgm.cnrs-giffr/download/ fasta/assemblies/): ptetraurelia_mac_51.fa and ptetraurelia_mac_51_with_ies.fa. The score for each IES corresponds to the mean of the two boundary scores.

\section{Assembly of MIC reads}

The MIC flow cytometry sequencing reads (acc. no. SAMN05323660; Additional file 2: Table S1) were assembled into contigs using ParTIES v1.0 [11] (default parameters except for the Assembly module, -k 51). ParTIES filters out reads that contain a MAC IES junction using the MAC reference genome prior to a Velvet (version 1.2.10) [51] assembly. Assembly statistics for the resulting MIC contigs are given in (Additional file 2: Table S2).

\section{Analysis of depth}

The MIC contigs (Additional file 2: Table S2) were used as reference genome for this analysis. The contigs were divided into $1-\mathrm{kb}$ non-overlapping windows. For each sequencing sample, the mean depth for each window was calculated with Samtools [52] depth (v0.1.18 -q $30-\mathrm{Q} 30)$ on Bowtie 2 [53] (v2.2.3 -local $-\times$ 800) mappings. The mean depth was normalized according to the number of nucleotides sequenced in the sample, after excluding reads which match known contaminants (mitochondrial DNA, rDNA, bacterial genomes).

\section{Sequencing simulation}

We simulated sequencing data using ART version 2.3.7 [54] (--noALN -len 100 -seqSys HS10 -qShift 90 -qShift2 90 -mflen 300 -sdev 100). We specifed coverage using the fcov parameter, to obtain final coverage of $100 \times$. Thus, to obtain a dataset with $40 \%$ enrichment in MIC sequences, we simulated $40 \times$ coverage on the MIC assembly and $60 \times$ coverage on the MAC assembly and pooled the simulated reads. The analysis of depth was applied to the simulated read datasets.

\section{Differential coverage analysis}

DESeq2 software [55] was designed for differential analysis of NGS count data, and is typically used for gene expression studies i.e. to compare RNA-Seq read counts for genes across experimental conditions. We used DESeq2 (v. 1.14.0) to compare DNA-Seq read counts for non-overlapping MIC windows ( $1 \mathrm{~kb}$ windows and $>400 \mathrm{bp}$ windows at contig ends) across samples. For each sample (Additional file 2: Table S1), we provide to DESeq2 the number of uniquely mapping reads in each window. We considered windows with a fold-change $>2$ between MIC and other samples and an adj. $p$-value $<0.05$ to be differentially covered. Barplots (Fig. 6c) used the normalized counts determined for each sample by DESeq2.

\section{Sequence properties}

For selected windows (see text and Table 2), tandem repeats (micro- and mini-satellite) were identified using Tandem Repeats Finder [56] (version 4.07b, TRF parame-

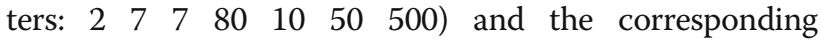
complexity determined using the $\mathrm{R}$ Bioconductor package "GenomicRanges_1.26.1" [57]. RepeatMasker [58] (version 3.3.0) was used to identify low complexity sequences (RepeatMasker -noint -no_is -s) and transposable elements (TE; RepeatMasker -nolow -no_is - $-\mathrm{s}-\mathrm{lib}<\mathrm{TE}$ consensus library>). The TE consensus library is that reported in this study (Additional file 3: Tables S3-S4). We performed exact binomial tests using the $\mathrm{R}$ package binom_1.1-1 [59].

\section{Additional files}

Additional file 1: This PDF contains the following supplementary figures: Figures S1- S7. Legends for these figures appear at the beginning of Additional file 1 (PDF $12230 \mathrm{~kb}$ )

Additional file 2: This word file contains the following supplementary tables: Tables S1, S2, S5, S6. (DOCX $110 \mathrm{~kb}$ )

Additional file 3: This excel file contains the following supplementary tables: Tables S3-S4. (XLSX 45 kb)

Additional file 4: This text file contains the following supplementary text: Text S1-S2. Text S1 is a fasta file of TE consensus nucleotide sequences. Text S2 is a fasta file of putative TE protein sequences. (TXT $280 \mathrm{~kb}$ )

\section{Abbreviations \\ APE: Apurinic/apyrimidinic endonuclease; FSC: Forward-scattered light; GFP: Green fluorescent protein; IES: Internal Eliminated Sequence; ITm: IS630-Tc1-mariner; MAC: Macronucleus; MIC: Micronucleus; RT: Reverse transcriptase; SSC: Side-scattered light; TE: Transposable element}

\section{Acknowledgements}

We thank Jean Cohen for his advice during the development of the purification procedure. We thank Isadora Cohen for critical reading of the manuscript and the SD lab members for support and discussion. We acknowledge the ImagoSeine facility, member of the France Biolmaging infrastructure supported by the ANR-10-INSB-04. The sequencing benefited from the facilities and expertise of the high-throughput sequencing platform of $12 B C$.

\section{Funding}

This research was supported by intramural funding from the CNRS, grant ANR-12-BSV6-0017 "INFERNO" to S. D., L. S. and E.M., program "Investissements $\mathrm{d}^{\prime}$ Avenir" launched by the French government and implemented by ANR with the references ANR-10-LABX-54 MEMOLIFE and ANR-11-IDEX-0001-02 PSL Research University, an 'Equipe FRM DEQ20150331763' grant to EM, grant ANR-14-CE10-0005-04 'PIGGYPACK' to S. D. and L. S., grants from LABEX "Who am I?" grant supported by the ANR-11-LABX-0071_WHOAMI and the 
ANR-11-IDEX-0005-02, from 'Comité d'lle de France de la Ligue Nationale Contre le Cancer', and an 'Equipe FRM DEQ20160334868' grant to S. D.

The funding bodies had no role in the design of the study, analysis, and interpretation of data and in writing the manuscript.

\section{Availability of data and materials}

All data generated or analysed during this study are listed in (Additional file 2: Table S1) (accession numbers to DNAseq datasets) or included in this published article and its supplementary information files.

\section{Authors' contributions}

FG performed all the experiments. NG carried out the sorting experiments. $\mathrm{OA}, \mathrm{CDW}$ and LS performed the bioinformatic analyses. EM identified and annotated transposable elements. SD conceived the study and wrote the paper. All authors read and approved the final manuscript.

\section{Competing interests}

The authors declare that they have no competing interests.

\section{Consent for publication}

Not applicable.

\section{Ethics approval and consent to participate}

Not applicable.

\section{Dedication}

We dedicate this work to the late John R. Preer Jr., who, with his wife Bertie, pioneered Paramecium nuclear purification and germline DNA characterization more than twenty years ago. John passed away in April 2016.

\section{Publisher's Note}

Springer Nature remains neutral with regard to jurisdictional claims in published maps and institutional affiliations.

\section{Author details}

'Institut Jacques Monod, CNRS, UMR 7592, Université Paris Diderot, Sorbonne Paris Cité, Paris F-75205, France. ${ }^{2}$ Institute of Integrative Biology of the Cell, UMR9198 CNRS CEA Univ, Paris-Sud Université Paris-Saclay, 91198 Gif-sur-Yvette, France. ${ }^{3}$ Current address: Institut de Biologie et de Technologies de Saclay (IBITECS), CEA, F-91191 Gif-sur-Yvette Cedex, France. ${ }^{4}$ IBENS, Département de Biologie, Ecole Normale Supérieure, CNRS, Inserm, PSL Research University, F-75005 Paris, France.

\section{Received: 24 December 2016 Accepted: 20 April 2017}

Published online: 26 April 2017

\section{References}

1. Wang J, Davis RE. Programmed DNA elimination in multicellular organisms. Curr Opin Genet Dev. 2014;27C:26-34.

2. Betermier M, Duharcourt S. Programmed Rearrangement in Ciliates: Paramecium. Microbiol Spectr. 2014;2.

3. Arnaiz O, Mathy N, Baudry C, Malinsky S, Aury J-M, Wilkes CD, et al. The Paramecium germline genome provides a niche for intragenic parasitic DNA: evolutionary dynamics of internal eliminated sequences. PLoS Genet. 2012;8:e1002984.

4. Holoch D, Moazed D. RNA-mediated epigenetic regulation of gene expression. Nat Rev Genet. 2015;16:71-84.

5. Coyne RS, Lhuillier-Akakpo M, Duharcourt S. RNA-guided DNA rearrangements in ciliates: Is the best genome defence a good offence? Biol Cell. 2012;104:1-17.

6. Aury JM, Jaillon O, Duret L, Noel B, Jubin C, Porcel BM, et al. Global trends of whole-genome duplications revealed by the ciliate Paramecium tetraurelia. Nature. 2006:444:171-8.

7. Preer LB, Hamilton G, Preer JR. Micronuclear DNA from Paramecium tetraurelia: serotype 51 A gene has internally eliminated sequences. J Protozool. 1992;39:678-82.

8. Steele CJ, Barkocy-Gallagher GA, Preer LB, Preer JR. Developmentally excised sequences in micronuclear DNA of Paramecium. Proc Natl Acad Sci U S A. 1994;91:2255-9.

9. Baudry C, Malinsky S, Restituito M, Kapusta A, Rosa S, Meyer E, et al. PiggyMac, a domesticated piggyBac transposase involved in programmed genome rearrangements in the ciliate Paramecium tetraurelia. Genes Dev. 2009;23:2478-83.

10. Berger JD. Selective inhibition of DNA synthesis in macronuclear fragments in Paramecium aurelia exconjugants and its reversal during macronuclear regeneration. Chromosoma. 1973;44:33-48.

11. Denby Wilkes C, Arnaiz O, Sperling L. ParTIES: a toolbox for Paramecium interspersed DNA elimination studies. Bioinforma Oxf Engl. 2016;32:599-601.

12. Chen X, Bracht JR, Goldman AD, Dolzhenko E, Clay DM, Swart EC, et al. The architecture of a scrambled genome reveals massive levels of genomic rearrangement during development. Cell. 2014;158:1187-98.

13. Fass JN, Joshi NA, Couvillion MT, Bowen J, Gorovsky MA, Hamilton EP, et al. Genome-Scale Analysis of Programmed DNA Elimination Sites in Tetrahymena thermophila. G3 Bethesda Md. 2011;1:515-22.

14. Hamilton EP, Kapusta A, Huvos PE, Bidwell SL, Zafar N, Tang H, et al. Structure of the germline genome of Tetrahymenathermophila and relationship to the massively rearranged somatic genome. elife. 2016;5.

15. Lhuillier-Akakpo M, Guérin F, Frapporti A, Duharcourt S. DNA deletion as a mechanism for developmentally programmed centromere loss. Nucleic Acids Res. 2016:44:1553-65.

16. Le Mouel A, Butler A, Caron F, Meyer E. Developmentally regulated chromosome fragmentation linked to imprecise elimination of repeated sequences in paramecia. Eukaryot Cell. 2003;2:1076-90.

17. Wicker T, Sabot F, Hua-Van A, Bennetzen JL, Capy P, Chalhoub B, et al. A unified classification system for eukaryotic transposable elements. Nat Rev Genet. 2007;8:973-82.

18. Malik HS, Burke WD, Eickbush TH. The age and evolution of non-LTR retrotransposable elements. Mol Biol Evol. 1999;16:793-805.

19. Fillingham JS, Thing TA, Vythilingum N, Keuroghlian A, Bruno D, Golding GB, et al. A Non-Long Terminal Repeat Retrotransposon Family Is Restricted to the Germ Line Micronucleus of the Ciliated Protozoan Tetrahymena thermophila. Eukaryot Cell. 2004;3:157-69.

20. Tay WT, Behere GT, Batterham P, Heckel DG. Generation of microsatellite repeat families by RTE retrotransposons in lepidopteran genomes. BMC Evol Biol. 2010;10:144.

21. Grandi FC, An W. Non-LTR retrotransposons and microsatellites. Mob Genet Elem. 2013;3, e25674.

22. Shao H, Tu Z. Expanding the Diversity of the IS630-TC1-mariner Superfamily: Discovery of a Unique DD37E Transposon and Reclassification of the DD37D and DD39D Transposons. Genetics. 2001;159:1103-15.

23. Herrick G, Cartinhour S, Dawson D, Ang D, Sheets R, Lee A, et al. Mobile elements bounded by C4A4 telomeric repeats in oxytricha fallax. Cell. 1985;43:759-68.

24. Jaraczewski JW, Frels JS, Jahn CL. Developmentally regulated, low abundance Tec element transcripts in Euplotes crassus-implications for DNA elimination and transposition. Nucleic Acids Res. 1994;22:4535.

25. Doak TG, Witherspoon DJ, Jahn CL, Herrick G. Selection on the genes of Euplotes crassus Tec1 and Tec2 transposons: evolutionary appearance of a programmed frameshift in a Tec2 gene encoding a tyrosine family site-specific recombinase. Eukaryot Cell. 2003;2:95-102.

26. Brillet B, Bigot Y, Augé-Gouillou C. Assembly of the Tc1 and mariner transposition initiation complexes depends on the origins of their transposase DNA binding domains. Genetica. 2007;130:105-20.

27. Lhuillier-Akakpo M, Frapporti A, Denby Wilkes C, Matelot M, Vervoort M, Sperling $L$, et al. Local effect of enhancer of zeste-like reveals cooperation of epigenetic and cis-acting determinants for zygotic genome rearrangements. PLoS Genet. 2014;10, e1004665.

28. Macas J, Lambert GM, Dolezel D, Galbraith DW. Nuclear expressed sequence Tag (NEST) analysis: a novel means to study transcription through amplification of nuclear RNA. Cytometry. 1998;33:460-8.

29. Borges F, Gardner R, Lopes T, Calarco JP, Boavida LC, Slotkin RK, et al. FACS-based purification of Arabidopsis microspores, sperm cells and vegetative nuclei. Plant Methods. 2012:8:44.

30. Samadder P, Weng N, Doetschman T, Heimark RL, Galbraith DW. Flow cytometry and single nucleus sorting for Cre-based analysis of changes in transcriptional states. Cytometry A. 2016;89:430-42.

31. Zhang C, Barthelson RA, Lambert GM, Galbraith DW. Global characterization of cell-specific gene expression through fluorescence-activated sorting of nuclei. Plant Physiol. 2008;147:30-40.

32. Marion-Poll L, Montalban E, Munier A, Hervé D, Girault J-A. Fluorescenceactivated sorting of fixed nuclei: a general method for studying nuclei from specific cell populations that preserves post-translational modifications. Eur J Neurosci. 2014;39:1234-44. 
33. Haenni S, Ji Z, Hoque M, Rust N, Sharpe H, Eberhard R, et al. Analysis of C. elegans intestinal gene expression and polyadenylation by fluorescenceactivated nuclei sorting and 3'-end-seq. Nucleic Acids Res. 2012;40:6304-18.

34. Bushman DM, Kaeser GE, Siddoway B, Westra JW, Rivera RR, Rehen SK, et al. Genomic mosaicism with increased amyloid precursor protein (APP) gene copy number in single neurons from sporadic Alzheimer's disease brains. elife. 2015;4.

35. Schoft VK, Chumak N, Bindics J, Slusarz L, Twell D, Köhler C, et al. SYBR Green-activated sorting of Arabidopsis pollen nuclei based on different DNA/RNA content. Plant Reprod. 2015;28:61-72.

36. Lake BB, Ai R, Kaeser GE, Salathia NS, Yung YC, Liu R, et al. Neuronal subtypes and diversity revealed by single-nucleus RNA sequencing of the human brain. Science. 2016;352:1586-90.

37. Okada S, Saiwai H, Kumamaru H, Kubota K, Harada A, Yamaguchi M, et al. Flow cytometric sorting of neuronal and glial nuclei from central nervous system tissue. J Cell Physiol. 2011;226:552-8.

38. Lacar B, Linker SB, Jaeger BN, Krishnaswami S, Barron J, Kelder M, et al. Nuclear RNA-seq of single neurons reveals molecular signatures of activation. Nat Commun. 2016;7:11022.

39. Krishnaswami SR, Grindberg RV, Novotny M, Venepally P, Lacar B, Bhutani K, et al. Using single nuclei for RNA-seq to capture the transcriptome of postmortem neurons. Nat Protoc. 2016;11:499-524.

40. Wiedenheft B, Sternberg SH, Doudna JA. RNA-guided genetic silencing systems in bacteria and archaea. Nature. 2012:482:331-8.

41. Galvani A, Sperling L. RNA interference by feeding in Paramecium. Trends Genet. 2002;18:11-2

42. Timmons L, Court DL, Fire A. Ingestion of bacterially expressed dsRNAs can produce specific and potent genetic interference in Caenorhabditis elegans. Gene. 2001;263:103-12.

43. Timmons L, Fire A. Specific interference by ingested dsRNA. Nature. 1998;395:854

44. Creutz CE, Mohanty S, Defalco T, Kretsinger RH. Purine composition of the crystalline cytoplasmic inclusions of Paramecium tetraurelia. Protist. 2002; 153:39-45.

45. Edgar RC. MUSCLE: multiple sequence alignment with high accuracy and high throughput. Nucleic Acids Res. 2004;32:1792-7.

46. Liu Y, Schmidt B, Maskell DL. MSAProbs: multiple sequence alignment based on pair hidden Markov models and partition function posterior probabilities. Bioinformatics. 2010;26:1958-64.

47. Guindon S, Gascuel O. A simple, fast, and accurate algorithm to estimate large phylogenies by maximum likelihood. Syst Biol. 2003;52:696-704.

48. Guindon S, Dufayard J-F, Lefort V, Anisimova M, Hordijk W, Gascuel O. New Algorithms and Methods to Estimate Maximum-Likelihood Phylogenies: Assessing the Performance of PhyML 3.0. Syst. Biol. 2010;59:307-21.

49. Stöver BC, Müller KF. TreeGraph 2: Combining and visualizing evidence from different phylogenetic analyses. BMC Bioinformatics. 2010;11:7.

50. Gouy M, Guindon S, Gascuel O. SeaView Version 4: A Multiplatform Graphical User Interface for Sequence Alignment and Phylogenetic Tree Building. Mol Biol Evol. 2010;27:221-4.

51. Zerbino DR, Birney E. Velvet: algorithms for de novo short read assembly using de Bruijn graphs. Genome Res. 2008:18:821-9.

52. Li H, Handsaker B, Wysoker A, Fennell T, Ruan J, Homer N, et al. The Sequence Alignment/Map format and SAMtools. Bioinforma Oxf Engl. 2009; 25:2078-9.

53. Langmead B, Salzberg SL. Fast gapped-read alignment with Bowtie 2. Nat Methods. 2012;9:357-9.

54. Huang W, Li L, Myers JR, Marth GT. ART: a next-generation sequencing read simulator. Bioinforma Oxf Engl. 2012;28:593-4.

55. Love Ml, Huber W, Anders S. Moderated estimation of fold change and dispersion for RNA-seq data with DESeq2. Genome Biol. 2014;15:550.

56. Benson G. Tandem repeats finder: a program to analyze DNA sequences. Nucleic Acids Res. 1999;27:573-80.

57. Lawrence $M$, Huber $W$, Pagès $H$, Aboyoun $P$, Carlson $M$, Gentleman $R$, et al. Software for computing and annotating genomic ranges. PLoS Comput Biol. 2013;9, e1003118.

58. Smit A, Hubley R, Green P. RepeatMasker Open-4.0. 2013. http://www. repeatmasker.org/.

59. Dorai-Raj S. binom: Binomial Confidence Intervals For Several Parameterizations. R package version 1.0-5. 2009. http://CRAN.R-project.org/ package $=$ binom

\section{Submit your next manuscript to BioMed Central and we will help you at every step:}

- We accept pre-submission inquiries

- Our selector tool helps you to find the most relevant journal

- We provide round the clock customer support

- Convenient online submission

- Thorough peer review

- Inclusion in PubMed and all major indexing services

- Maximum visibility for your research

Submit your manuscript at www.biomedcentral.com/submit 